\title{
Determinants of the Glomerular Maximal Size Threshold
}

\author{
Nobuo Tsuboi \\ Division of Nephrology and Hypertension, Department of Internal Medicine, The Jikei University School of Medicine, \\ Tokyo, Japan
}

Kidney biopsy is an established standard approach for the histopathological diagnosis of kidney diseases, with additional information also available for determining a therapeutic intervention strategy and predicting kidney disease outcomes. Thus far, biopsy specimens have been used for various morphometric analyses of the kidneys, mainly for research purposes [1-4]. The most widely accepted approach is measuring the size of the glomeruli, which can be regarded as morphologically almost spherical and allow the use of any section of the obtained biopsy materials. Using biopsy materials, previous studies have measured indices of glomerular size, such as the glomerular diameter, glomerular cross-sectional area, and estimated glomerular volume. Other studies used kidney biopsy specimens to estimate the glomerular density per area as a surrogate of the total nephron number [5]. Further, a recent new approach enabled the estimation of the total nephron number per kidney by the combination of the assessment of the cortical volume on the CT image and stereology-based measurement of the volumetric glomerular density in biopsy specimens [6]. The major objective of these morphometric approaches for estimating the glomerular number or size in a clinical setting is to elucidate the clinical-histological correlations during the progressive loss of functioning nephrons, in which glomerular hypertrophy may occur as a surrogate of glomerular hyperfiltration/hypertension [7]. In fact, glomerular enlargement in diagnostic kidney biopsy has been consistently identified as a hallmark of worse kidney outcomes in a series of etiologically different kidney diseases in previous studies [1-3]. A previous study in patients with diabetes has shown that glomerular size may determine the rate of progression of the loss of kidney function in subjects destined to develop diabetic nephropathy [4].

Studies have suggested some factors that may be essential for the determination of glomerular size variability between individuals. A study that compared various kidney morphometric indices in autopsies of children and adults has clearly shown a close relationship between body size and glomerular size [8]. The difference in glomerular size between children and adults with similar total nephron counts was approximately 6 -fold, corresponding to the body size difference. A correlation between body size and glomerular size has been consistently identified in several biopsy-based studies, and a typical histopathological characteristic in proteinuric glomerulopathy seen in obese individuals is markedly enlarged
C 2021 The Author(s).

Published by S. Karger AG, Basel

This is an Open Access article licensed under the Creative Commons Attribution-NonCommercial-4.0 International License (CC BY-NC) (http://www.karger.com/Services/OpenAccessLicense), applicable to the online version of the article only. Usage and distribution for commercial purposes requires written permission.
Correspondence to:

Nobuo Tsuboi, tsuboi-n@jikei.ac.jp 
Fig. 1. Factors that may determine the consequences of the glomerular hypertrophic response to injuries. Various systemic or intrarenal local factors may determine the consequences of the glomerular hypertrophic response to injuries. These include whole-body metabolic demand, kidney structural or numerical diversity (nephrons/podocytes) between individuals, acquired factors, such as aging/comorbidities or specific glomerulopathies, and the balance between glomerular hypertension and vulnerability. Depending on the number and severity of these determinants, the compensatory enlargement of the glomerulus remains or a decompensatory state results, which leads to glomerulosclerosis. DM, diabetes mellitus; HTN, hypertension; IgAN, immunoglobulin A nephropathy.

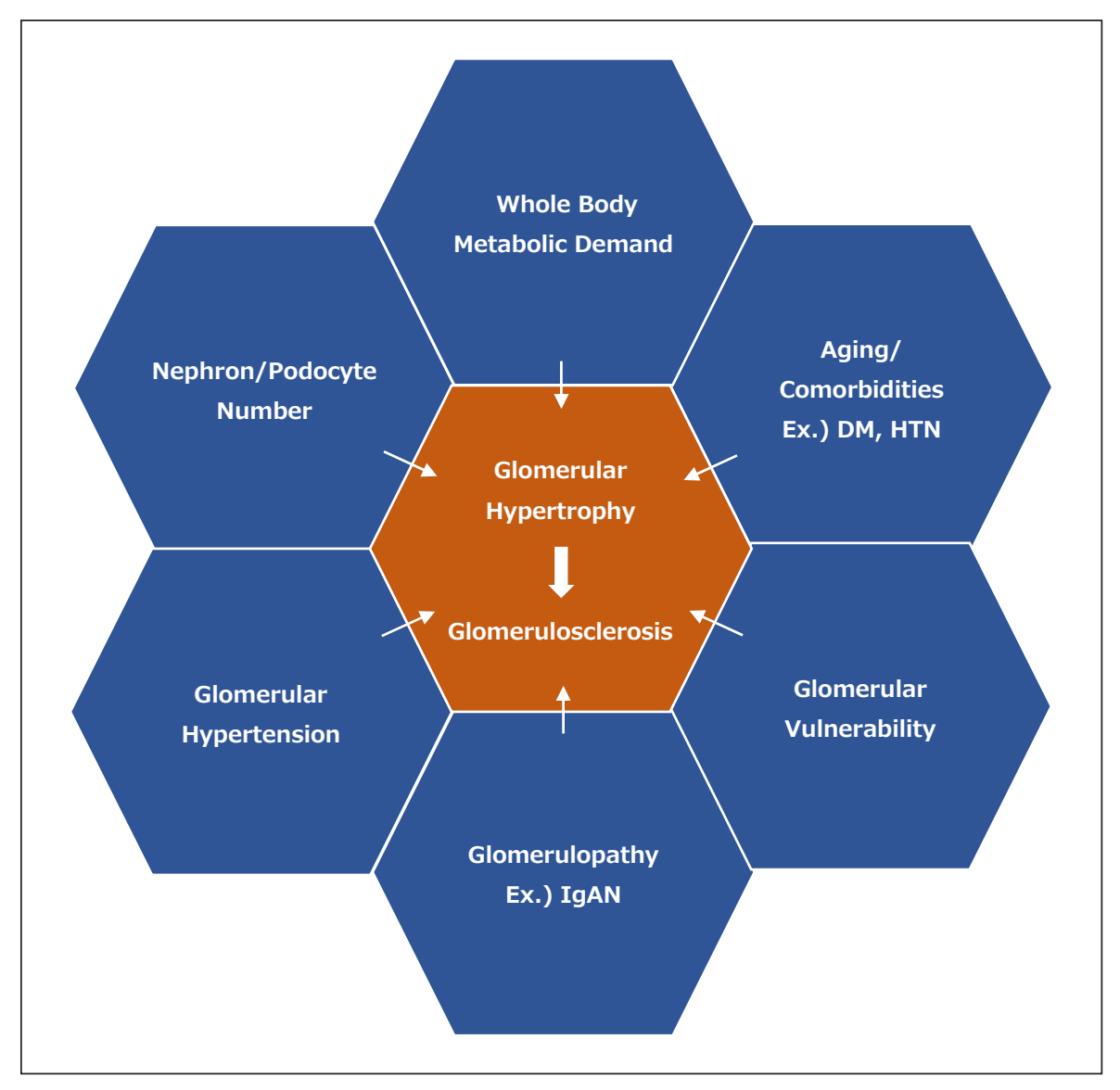

glomeruli, called glomerulomegaly [9]. These findings suggest that whole-body metabolic demand due to a body size increase is primarily an important determinant of glomerular size in both healthy and/or diseased conditions. Second, studies on autopsy series without apparent kidney diseases have disclosed that the human nephron number varies significantly between individuals and is inversely correlated with glomerular size [10]. Consistent with the hypothesis of glomerular adaptation failure raised by experimental animal studies using a kidney ablation model [7], the innate or acquired reduction of the nephron number has been linked to enlarged glomerular size in many studies [10]. The third factor is the number of glomerular podocytes. Glomerular podocytes are highly differentiated cells with a limited capacity to divide or regenerate and are essential to maintaining the structure and filtration of the glomerulus. The detachment of podocytes due to failure to cover the glomerular surface may constitute a pathway to glomerular filtration failure, which is closely associated with the appearance of focal segmental glomerulosclerosis (FSGS) lesions as a result of glomerular scarring [11]. Of note, a recent study showed that the number of glomerular podocytes varies significantly between individuals and is progressively lost with normal aging [12]. The number of glomerular podocytes may therefore limit the glomerular hypertrophic response and determine the maximal glomerular size threshold.

An overriding question in glomerular hypertrophy is the extent to which the glomerulus can be enlarged in size without losing the filtration function? In this issue of Kidney and Blood Pressure Research, Zamami et al. [13] raised and attempted to answer this important question. They measured the maximal glomerular diameter (max GD) as a surrogate of glomerular hypertrophy in patients with biopsy-diagnosed kidney diseases and cross-sectionally examined correlations between clinical and histopathological findings. The main outcome measure in this study was the appearance of FSGS lesions in the same biopsy specimens, which was regarded as a state of glomerular adaptation failure (partial loss of the glomerular filtration function) due to the excessive enlargement of the glomeruli. Consistent with their hypothesis, the authors dem- 
onstrated that max GD was independently associated with the appearance of FSGS lesions in multivariable analyses. The BMI did not show an independent association with FSGS lesions in multivariable models, suggesting that glomerular size was more closely related to FSGS lesions than to body size in the study population. Further, this study found that a max GD of $>224 \mu \mathrm{m}$ was the threshold value that was associated with the appearance of FSGS lesions. Interestingly, this threshold was similar to that determined in a previous study of Japanese patients for predicting worse kidney outcomes in patients with IgA nephropathy, which was $>242 \mu \mathrm{m}$ [3]. Several systemic and local factors may determine the consequence of the glomerular hypertrophic response to injuries, depending on number and severity of these factors (Fig. 1). Importantly, the study by Zamami et al. [13] analyzed the biopsy specimens of CKD patients with various degrees of active and/or chronic glomerular lesions. Previous animal studies have suggested that the structural diversity of the kidneys, such as reductions in the nephron number or the number of podocytes per kidney, may further produce differences in susceptibility to glomerular injuries and progressive loss of the kidney function $[14,15]$. A previous study using an animal model of glomerulonephritis showed that an increase in the difference in transcapillary hydraulic pressure may compensate for a decrease in the glomerular capillary ultrafiltration coefficient, possibly to preserve single-nephron glomerular filtration [16]. These findings suggest that a balance between the glomerular hypertrophic response and glomerular vulnerability is an important determinant for the consequence of the glomerular response to injuries. The finding of an association between max GD and the appearance of secondary FSGS lesions, in the presence human glomerulopathies - as reported by Zamami et al. [13] - may therefore provide invaluable information for considering approaches to the treatment of patients with progressive kidney disease in the clinical setting. Further validation of the maximum glomerular size threshold associated with the appearance of glomerulosclerosis identified in this study may pave the way to elucidate the pathophysiological mechanisms involved in a common pathway of progression in CKD.

\section{Conflict of Interest Statement}

The author has no conflicts of interest to declare.

\section{Funding Sources}

There is no funding source associated with this article.

\section{References}

1 Fogo A, Ichikawa I. Evidence for a pathogenic linkage between glomerular hypertrophy and sclerosis. Am J Kidney Dis. 1991;17(6): 666-9.

2 Nishimoto K, Shiiki H, Nishino T, Uyama H, Iwano M, Dohi K. Reversible glomerular hypertrophy in adult patients with primary focal segmental glomerulosclerosis. J Am Soc Nephrol. 1997;8(11):1668-78.

3 Tsuboi N, Kawamura T, Ishii T, Utsunomiya $\mathrm{Y}, \mathrm{Hosoya} \mathrm{T}$. Changes in the glomerular density and size in serial renal biopsies during the progression of IgA nephropathy. Nephrol Dial Transplant. 2009;24(3):892-9.

4 Bilous RW, Mauer SM, Sutherland DE, Steffes MW. Mean glomerular volume and rate of development of diabetic nephropathy. Diabetes. 1989;38(9):1142-7.

5 Kataoka H, Ohara M, Honda K, Mochizuki T, Nitta K. Maximal glomerular diameter as a 10 -year prognostic indicator for IgA nephropathy. Nephrol Dial Transplant. 2011; 26(12):3937-43.

6 Denic A, Mathew J, Lerman LO, Lieske JC, Larson JJ, Alexander MP, et al. Single-nephron glomerular filtration rate in healthy adults. N Engl J Med. 2017 15;376(24):2349-57.
7 Hostetter TH, Olson JL, Rennke HG, Venkatachalam MA, Brenner BM. Hyperfiltration in remnant nephrons: a potentially adverse response to renal ablation. Am J Physiol. 1981;241(1):F85-93.

8 Puelles VG, Douglas-Denton RN, CullenMcEwen LA, Li J, Hughson MD, Hoy WE, et al. Podocyte number in children and adults: associations with glomerular size and numbers of other glomerular resident cells. J Am Soc Nephrol. 2015;26(9):227788.

9 Tsuboi N, Okabayashi Y, Shimizu A, Yokoo T. The renal pathology of obesity. Kidney Int Rep. 2017 Jan 23;2(2):251-60.

10 Nyengaard JR, Bendtsen TF. Glomerular number and size in relation to age, kidney weight, and body surface in normal man. Anat Rec. 1992;232:194-201.

11 Hodgin JB, Bitzer M, Wickman L, Afshinnia F, Wang SQ, O'Connor C, et al. Glomerular aging and focal global glomerulosclerosis: a podometric perspective. J Am Soc Nephrol. 2015;26(12):3162-78.
12 Haruhara K, Sasaki T, de Zoysa N, Okabayashi Y, Kanzaki G, Yamamoto I, et al. Podometrics in Japanese living donor kidneys: associations with nephron number, age, and hypertension. J Am Soc Nephrol. 2021;32(5): 1187-99. ASN.2020101486.

13 Zamami, Kohagura K, Kinjo K, Nakamura T, Kinjo T, Yamazato M, et al. Association between glomerular diameter and secondary focal segmental glomerulosclerosis in chronic kidney disease. Kidney Blood Pres Res. doi: $10.1159 / 000515528$.

14 Cheng QL, Orikasa M, Morioka T, Kawachi $\mathrm{H}$, Chen XM, Oite T, et al. Progressive renal lesions induced by administration of monoclonal antibody $1-22-3$ to unilaterally nephrectomized rats. Clin Exp Immunol. 1995; 102(1):181-5.

15 Matsusaka T, Sandgren E, Shintani A, Kon V, Pastan I, Fogo AB, et al. Podocyte injury damages other podocytes. J Am Soc Nephrol. 2011;22(7):1275-85.

16 Maddox DA, Bennett CM, Deen WM, Glassock RJ, Knutson D, Daugharty TM, et al. Determinants of glomerular filtration in experimental glomerulonephritis in the rat. J Clin Invest. 1975;55(2):305-18. 